\title{
Prohibitin and the extracellular matrix are upregulated in murine alveolar epithelial cells with LPS-induced acute injury
}

\author{
FENG ZHANG $^{1,2}$, DEJUN FAN ${ }^{3}$ and XIAO-NENG MO ${ }^{1,4}$ \\ Departments of ${ }^{1}$ Respiratory Medicine, ${ }^{2}$ Rheumatology and ${ }^{3}$ Gastrointestinal Endoscopy, \\ The Sixth Affiliated Hospital, Sun Yat-sen University, Guangzhou, Guangdong 510655; ${ }^{4}$ Department of \\ Respiratory Medicine, No. 8 People's Hospital of Guangzhou, Guangzhou, Guangdong 510060, P.R. China
}

Received March 19, 2017; Accepted November 23, 2017

DOI: $10.3892 / \mathrm{mmr} .2018 .8808$

\begin{abstract}
Inflammation of epithelial and endothelial cells accelerates the progress of acute lung injury (ALI), and pulmonary fibrosis is the leading cause of mortality in patients with acute respiratory distress syndrome. Interleukin-6 (IL-6) is a pleiotropic cytokine implicated in the pathogenesis of a number of immune-mediated disorders, and is involved in pulmonary fibrosis. Prohibitin (PHB) is a highly conserved protein implicated in various cellular functions, including proliferation, apoptosis, tumor suppression, transcription and mitochondrial protein folding. PHB was identified to be associated with a variety of pulmonary diseases, including pulmonary fibrosis. Based on the lipopolysaccharide (LPS)-induced cell model of ALI, the present study examined the expression of PHB and the extracellular matrix (ECM) in the process of pulmonary inflammation. MLE-12 cells were divided into 2 groups: The control group was administered sterile PBS; the treatment group was administered $500 \mathrm{ng} / \mathrm{ml}$ LPS for $12 \mathrm{~h}$. The mRNA expression of IL-6 in the treatment group was significantly upregulated compared with the control group $(\mathrm{P}<0.05)$. The protein expression of IL- 6 in the treatment group was markedly increased compared with the control group $(\mathrm{P}<0.05)$. ECM components, including collagen-IV and fibronectin, in the treatment group were markedly increased when compared with the control group $(\mathrm{P}<0.05)$. The mRNA and protein expression levels of PHB1 and $\mathrm{PHB} 2$ were significantly upregulated following treatment with LPS (both $\mathrm{P}<0.05$ ). The present study identified that PHB and ECM component levels increased in the LPS-induced ALI cell model, and further investigations may be performed to verify the detailed mechanism.
\end{abstract}

Correspondence to: Dr Xiao-Neng Mo, Department of Respiratory Medicine, The Sixth Affiliated Hospital, Sun Yat-sen University, 26 Yuancun Erheng Road, Guangzhou, Guangdong 510655, P.R. China E-mail: xiaonengmo100@126.com

Key words: acute lung injury, interleukin-6, prohibitin, extracellular matrix, murine alveolar epithelial cell

\section{Introduction}

Acute lung injury (ALI) is the early stage of acute respiratory distress syndrome (ARDS), which is a severe inflammatory injury to the lung (1). ALI/ARDS is characterized by the accumulation of protein-rich edema fluid in the alveolar compartments of the lung (2). The mechanism of lung injury varies with the cause; for example, increased vascular permeability, overproduction of cytokines, leukocyte recruitment and surfactant dysfunction, leading to interstitial and alveolar pulmonary edema, alveolar collapse and hypoxemia. Damage to epithelial and endothelial cells serves an important role in the development of ALI/ARDS (3). The clinical manifestation is characterized by regeneration and healing via resection or repair, frequently leading to persistent intra-alveolar and interstitial fibrosis (4). Survivors of ARDS are often exhibit chronic pulmonary fibrosis, reduced pulmonary function and diminished health-related quality of life (5). Indeed, chronic inflammation and tissue fibrosis are principal causes of morbidity and mortality in the chronic stage of ARDS, which is responsible for $\sim 30 \%$ of hospital mortalities following lung resection (6).

Pulmonary fibrosis is a devastating lung problem characterized by diffuse interstitial inflammation and exaggerated collagen accumulation, which in turn leads to the destruction of alveolar structures and remodeling (7). Extracellular matrix (ECM) accumulation includes the accumulation of collagen-IV (Col-IV) and fibronectin (FN). The pathogenesis of pulmonary fibrosis in acute lung injury, including ARDS, is poorly understood at present; therefore, there is an urgent need to expand the present understanding of the pathogenesis of ALI.

Interleukin (IL)-6 is a pleiotropic cytokine implicated in the pathogenesis of numerous infectious diseases and a number of immune-mediated disorders $(8,9)$. IL- 6 binds to an IL-6 receptor, and associates with a dimer of the ubiquitously-expressed gp130 receptor subunit, which initiates intracellular signaling (9). Certain studies have indicated that IL-6 is involved in pulmonary fibrosis in vivo (10-12).

Prohibitin (PHB) is a highly-conserved protein that has multiple functions in the cell (10-12). The PHB1 gene is located on chromosome 17q21 and encodes a $30 \mathrm{kDa}$ protein that associates with prohibitin 2 (PHB2) forming 16-20-mer 
ring-like structures with chaperone or scaffolding activities within the mitochondria (13). These structures share $>50 \%$ identical amino acid residues and are two members of the PHB family (14). PHB1, along with the highly homologous PHB2, is ubiquitously expressed in an array of eukaryotic organisms (15). PHB1 is involved in multiple cellular functions and the subcellular localization of prohibitin may determine its function (16). PHB in membranes regulates the cellular signaling of membrane transport, nuclear PHB controls transcription activation and the cell cycle, and the mitochondrial PHB complex stabilizes the mitochondrial genome and modulates mitochondrial dynamics, mitochondrial morphology, mitochondrial biogenesis and the mitochondrial intrinsic apoptotic pathway (16). Alterations in PHB1 levels have been associated with pathologies, including inflammation, obesity, autoimmunity or cancer (17-19). PHB1, which may be a potential therapeutic target for the treatment of a variety of diseases, has been reported to prevent inflammation-associated oxidative stress and injury due to its antioxidant properties $(20,21)$.

Previously, certain studies identified that PHB was associated with pulmonary disease. Soulitzis et al (22) reported that, non-chronic obstructive pulmonary disease (COPD) smokers exhibited lower PHB1 mRNA expression levels when compared with non-smokers, while PHB1 expression was even further decreased in patients with COPD. By contrast, PHB2 levels were similar among the three study groups. Agrawal et al (23) demonstrated that in a mouse model of allergic airway inflammation, vitamin D deficiency decreased the expression of vitamin D receptor (VDR) and PHB. Supplementation with vitamin D may increase the expression of VDR and PHB, which may be responsible for reducing allergic airway inflammation. Meanwhile, certain studies identified that PHB was associated with inflammation and fibrosis. PHB expression has been proven to be negatively correlated with hepatic, intestinal and renal interstitial fibrosis (24-26).

Lipopolysaccharide (LPS) is widely accepted in the establishment of ALI models and has the ability to induce the release of numerous inflammatory mediators, including tumor necrosis factor (TNF)- $\alpha$, IL-1 $\beta$, IL-6, NO and superoxide anions (27). Intratracheal administration of LPS increases cytokine levels in bronchial alveolar lavage fluids, whereas LPS challenge in lung endothelial or bronchial epithelial cells enhances barrier permeability and IL-6 release (28).

At present, to the best of our knowledge, there have been no studies confirming the roles served by PHB in ALI. Based on the LPS-induced cell model of ALI, the present study explored the expression of PHB and ECM in the process of ALI. The results of the present study revealed that PHB and ECM increased in an LPS-induced acute injury cell model.

\section{Materials and methods}

Cell culture and treatments. The murine alveolar epithelial cell line MLE-12 was purchased from the American Type Culture Collections (ATCC; Manassas, VA, USA) and cultured in Dulbecco's modified Eagle's medium/F12 supplemented with $10 \%$ fetal bovine serum (both from Gibco; Thermo Fisher Scientific, Inc., Waltham, MA, USA), $0.1 \mathrm{mg} / \mathrm{ml}$ streptomycin and $100 \mathrm{U} / \mathrm{ml}$ penicillin (Gibco; Thermo Fisher Scientific, Inc.) and maintained in a $5 \% \mathrm{CO}_{2}$ and $95 \%$ air atmosphere at $37^{\circ} \mathrm{C}$. The culture medium was replaced with fresh medium every 2 days. Cells were plated at a density of $4 \times 10^{5}$ cells/well in 6-well plates overnight. Once the cells reached $80 \%$ confluence, they were divided into two groups and treated as follows: The control group was treated with sterile PBS and the treatment group was stimulated with $500 \mathrm{ng} / \mathrm{ml}$ LPS (Sigma-Aldrich; Merck KGaA, Darmstadt, Germany) for $12 \mathrm{~h}$ (determined by our pre-experiment) (Zang et al, unpublished study). Total proteins and mRNA were extracted from cells following the $12 \mathrm{~h}$ incubation.

Reverse transcription-quantitative polymerase chain reaction (RT-qPCR) analysis. To detect the mRNA expression levels of IL-6, PHB1 and PHB2, total RNA was extracted from each group using TRIzol reagent (Invitrogen; Thermo Fisher Scientific, Inc.) according to the manufacturer's instructions, and the quality and concentration were assessed. RNA $(1 \mu \mathrm{g})$ was reverse-transcribed using the ReverTra Ace qPCR RT kit (cat. no. FSQ-101; Toyobo Co., Ltd., Osaka, Japan), according to the manufacturer's instructions. qPCR was performed with SYBR Green Real-time PCR Master Mix (Takara Bio, Inc., Otsu, Japan) on an Applied Biosystems 7500 Real-time PCR system (Applied Biosystems; Thermo Fisher Scientific, Inc.). The qPCR thermal cycling was performed as follows: Initial incubation for $10 \mathrm{~min}$ at $95^{\circ} \mathrm{C}$, followed by denaturing for 40 cycles at $95^{\circ} \mathrm{C}$ for $15 \mathrm{sec}$ and annealing for $60 \mathrm{sec}$ at $60^{\circ} \mathrm{C}$, and elongation at $60^{\circ} \mathrm{C}$ for $15 \mathrm{sec}$. All reactions were performed in triplicate, with three samples from different groups. The quantification of target mRNAs was normalized to $\beta$-actin, an internal control gene. The average quantification cycle (Cq; the cycles of template amplification to the threshold) was worked out as the value of each sample. The data for fold change was analyzed using the $2^{-\Delta \Delta \mathrm{Cq}}$ method (29). The primer sequences were as follows: $\beta$-actin forward, 5'-ATGGAGGG GAATACAGCCC-3' and reverse, 5'-TTCTTTGCAGCTCCT TCGTT-3'; PHB1 forward, 5'-GGGAAGGAGTTCACAGAG GCAGTA-3' and reverse, 5'-CACCCTCAGCAGAGATGA TGGC-3'; PHB2 forward, 5'-CAAGAACCCCACCACCAG AGAA-3' and reverse, 5'-TCCAAGAGGGCAGATACAGAA AAG-3'; IL-6 forward, 5'-ACCAGAGGAAATTTTCAATAG GC-3' and reverse, 5'-TGATGCACTTGCAGAAAACA-3'.

Western blot analysis. Protein was extracted using 1X SDS sample buffer (Sigma-Aldrich; Merck KGaA) with a cocktail of protease and phosphatase inhibitors. Following the measurement of protein concentration using a bicinchoninic acid kit (Beijing Dingguo Changsheng Biotechnology Co., Ltd., Beijing, China) with a microplate spectrophotometer (Thermo Fisher Scientific, Inc.), the protein samples were denatured by heating at $95^{\circ} \mathrm{C}$ for $10 \mathrm{~min}$, and $30 \mu \mathrm{g} /$ lane protein was separated by $9-15 \%$ SDS-PAGE (ranging according to the molecular weight of the target protein) and then transferred onto nitrocellulose membranes (EMD Millipore, Billerica, MA, USA). Membranes were blocked with 5\% non-fat milk in TBS with $0.1 \%$ Tween-20 (TBST; Sigma-Aldrich; Merck KGaA) at $4^{\circ} \mathrm{C}$ for $60 \mathrm{~min}$. Following rinsing with TBST, the membranes were probed with primary antibodies against PHB1 (cat. no. ab75771; 1:1,000), PHB2 (cat. no. ab154992; 1:1,000) (both from Abcam, Cambridge, UK), IL-6 (cat. no. 12,912; 1:1,000; Cell Signaling Technology, Inc., Danvers, MA, USA), Col-IV 
A

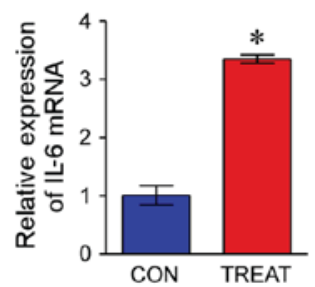

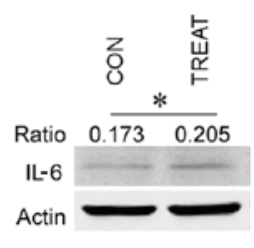

Figure 1. mRNA and protein expression of IL-6 in the murine alveolar epithelial cell line MLE12 is upregulated following stimulation with lipopolysaccharide. (A) Analysis of IL-6 mRNA expression by reverse transcription-qPCR. (B) Analysis of IL- 6 protein expression by western blotting. For qPCR, relative mRNA expression was normalized to GAPDH. For western blotting, $\beta$-actin was used as an internal control. Experiments were repeated at least three times with similar results. ${ }^{*} \mathrm{P}<0.05$ vs. control group. IL, interleukin; CON, control; TREAT, treatment group; qPCR, quantitative polymerase chain reaction.
A

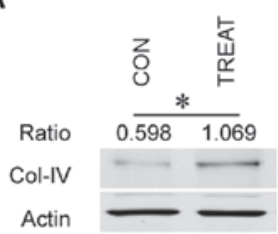

B

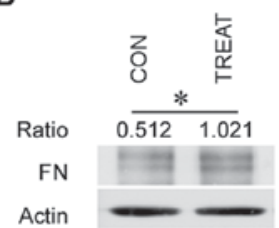

Figure 2. Extracellular matrix components, including Col-IV and FN, accumulate in murine alveolar epithelial cells following acute injury. (A) Analysis of Col-IV protein expression in MLE12 cells by western blotting. (B) Analysis of FN protein expression in MLE12 cells by western blotting. $\beta$-actin was used as an internal control in western blotting. Experiments were repeated at least three times with similar results. ${ }^{*} \mathrm{P}<0.05$ vs. control group. Col, collagen; FN, fibronectin; CON, control; TREAT, treatment group.

(cat. no. ab6586; 1:5,000) and FN (cat. no. ab45688; 1:5,000) (both from Abcam) at $4{ }^{\circ} \mathrm{C}$ overnight, followed by incubation with IRDye $800 \mathrm{CW}$ goat anti-rabbit secondary antibody (cat. no. 042-07-15-06; 1:10,000; LI-COR Biosciences, Lincoln, $\mathrm{NE}, \mathrm{USA}$ ) at $4{ }^{\circ} \mathrm{C}$ for $30 \mathrm{~min}$ protected from light. $\beta$-actin (cat. no. 4970; 1:1,000; Cell Signaling Technology, Inc.) was used as an internal loading control. Immunoblot detection was performed with the Odyssey Imaging System (LI-COR Biosciences) and densitometric analysis was performed using Adobe Photoshop (version 13.0; Adobe Systems Inc., San Jose, CA, USA).

Statistical analysis. Data are expressed as mean \pm standard deviation of $>3$ replicates. All statistical analyses were performed using SPSS version 19.0 statistical software (IBM Corp., Armonk, NY, USA). Comparisons between two groups were performed using the Student's t-test. $\mathrm{P}<0.05$ was considered to indicate a statistically significant difference.

\section{Results}

mRNA and IL-6 protein expression are upregulated following stimulation with LPS. The mRNA expression of IL-6 in MLE-12 following treatment with LPS (treatment group) was significantly upregulated compared with the control group $(\mathrm{P}<0.05$; Fig. 1A). Western blot analysis indicated that the protein expression of IL- 6 in the treatment group was markedly increased compared with the control group $(\mathrm{P}<0.05$; Fig. 1B).
A
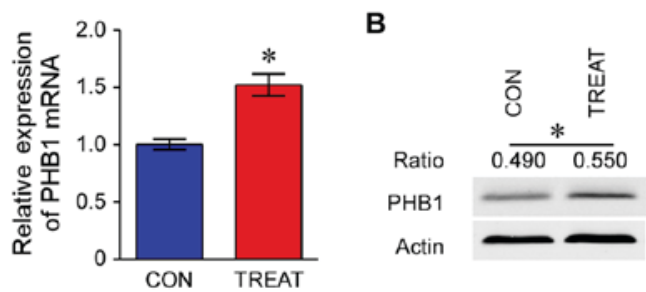

Figure 3. mRNA and protein expression of PHB1 in MLE12 cells is positively correlated with extracellular matrix accumulation. (A) Analysis of PHB1 mRNA expression by reverse transcription-qPCR. (B) Analysis of PHB1 protein by western blotting. For qPCR, relative expression of mRNA was normalized to GAPDH. For western blotting, $\beta$-actin was used as an internal control. Experiments were repeated at least three times with similar results. ${ }^{*} \mathrm{P}<0.05$ vs. control group. PHB, prohibitin; CON, control; TREAT, treatment group; qPCR, quantitative polymerase chain.

A

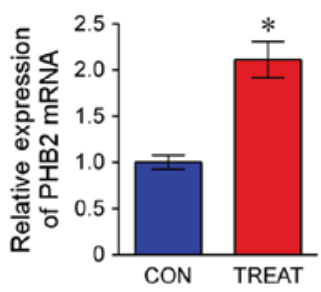

B

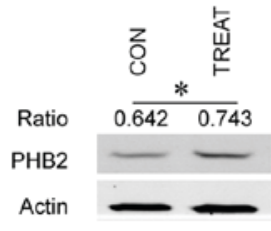

Figure 4. mRNA and protein expression of PHB2 in MLE12 is positively correlated with extracellular matrix accumulation. (A) Analysis of PHB2 mRNA expression by reverse transcription-qPCR. (B) Analysis of PHB2 protein by western blotting. For qPCR, relative expression of mRNA was normalized to GAPDH. For western blotting, $\beta$-actin was used as an internal control. Experiments were repeated at least three times with similar results. ${ }^{*} \mathrm{P}<0.05$ vs. control group. PHB, prohibitin; CON, control; TREAT, treatment group; qPCR, quantitative polymerase chain reaction.

ECM components Col-IV and FN accumulate in murine alveolar epithelial cells following acute injury. The protein expression levels of ECM components (Col-IV and FN) were detected via western blotting. ECM components, including Col-IV and FN, in the treatment group were markedly increased when compared with the control group $(\mathrm{P}<0.05$; Fig. 2$)$. This indicated that ECM components were accumulated in alveolar epithelial cells following acute inflammatory injury.

mRNA expression levels of PHB1 and PHB2, and western blot analysis of $P H B 1$ and $P H B 2$. The mRNA expression levels of PHB1 and PHB2 in the treatment group were significantly upregulated compared with the control group $(\mathrm{P}<0.05$; Figs. 3 and 4). Furthermore, western blot analysis indicated that protein expression levels of PHB1 and PHB2 in the treatment group were increased compared with the control group $(\mathrm{P}<0.05$; Figs. 3 and 4$)$.

\section{Discussion}

Pulmonary fibrosis is a chronic, progressive and irreversible process. Severe inflammatory injury in the lungs frequently leads to persistent fibrosis (4). Patients with ARDS frequently exhibit chronic pulmonary fibrosis. The presence of fibrosis is significantly correlated with the duration of ARDS. Additionally, fibrosis may be an important reason for the poor prognosis of patients with ARDS, since it leads to decreases in 
lung compliance and oxygenation (30,31). Pulmonary fibrosis is characterized by the activation of myofibroblasts, which may originate from epithelial cells via the onset of epithelial to mesenchymal transition (EMT). The persistence of the myofibroblasts beyond a period of normal repair has been associated with excessive ECM accumulation, finally resulting in pulmonary fibrosis $(32,33)$. A previous study demonstrated that LPS induces EMT and pulmonary fibrosis in vivo (34).

LPS, a major component of Gram-negative bacteria, is one of the principal pro-inflammatory reaction factors in infection diseases, leading to inflammatory overreactions in vitro and in vivo. It has been reported to be one of the primary factors that induces the inflammatory response, and the LPS-induced murine model of lung injury has been widely used to investigate the mechanisms of ALI $(28,35)$. In the model of ALI, LPS significantly increased the production of inflammatory cytokines including TNF- $\alpha$, IL- $1 \beta$ and IL-6, which has been demonstrated to be involved in the development of ALI (36). In the present study, a similar ALI cell model was created via stimulation with $500 \mathrm{ng} / \mathrm{ml} \mathrm{LPS}$, according to the protocol of Zhu et al (37). IL-6 was considered to be a marker of the LPS-induced inflammatory response (38). According to the data from the present study, the mRNA and protein expression levels of IL-6 were notably increased via LPS stimulation, compared with the control group. This finding was consistent with previous studies and indicated that the model of LPS-induced acute injury was successfully established.

A previous study (39) demonstrated that IL-6 response element (IL-6RE) is the essential transcription regulatory site for maximal basal and IL-6-induced PHB promoter activity. Signal transducer and activator of transcription 3 mediates basal and IL-6-induced PHB transcription and binds to IL-6RE in the PHB promoter. It may be hypothesized that IL-6 increases PHB protein and mRNA expression by activating the PHB promoter in vitro and in vivo (39). In the present study, the mRNA and protein expression levels of PHB1 and PHB2 in alveolar epithelial cells stimulated with LPS were increased compared with those in the control group. The present study revealed that increased IL- 6 was associated with the upregulation of PHB in murine alveolar epithelial cells.

Certain studies have identified that PHB is associated with inflammation and fibrosis in multiple diseases. Ko et al (24) suggested that a liver-specific deletion of PHB1 results in marked liver fibrosis. Yuan et al (40) reported that treatment with IL-10 is associated with increased PHB, and may decrease inflammation and fibrosis in an animal model of Crohn's disease; PHB may be a potential target for intestinal fibrosis associated with inflammatory bowel disease (IBD). Zhou et al (38) demonstrated that lower expression of PHB was associated with increased renal interstitial fibrosis and ECM accumulation. Previous studies $(20,21)$ suggested that PHB serves an important role in combating oxidative stress, by interacting with the NADH dehydrogenase subunits of mitochondrial respiratory complex I. Following the above studies, it was hypothesized that, in the LPS-induced ALI cell model, PHB may alleviate the process of pulmonary fibrosis due to its role in antioxidant stress.

In the present ALI model of alveolar epithelial cells, ECM and PHB were upregulated simultaneously. This appears to contradict the effect of PHB identified in previous reports on pulmonary disease and fibrosis, where PHB1 was proven to be a protective factor in patients with COPD (22) and the deletion of PHB1 was demonstrated to exacerbate liver fibrosis (24). The present study suggested that this discrepancy may be attributed to the protective effect of PHB, which may not reverse the process of LPS-induced fibrosis.

In a previous study of IBD, decreased expression of prohibitin was associated with intestinal fibrosis progression, and treatment with IL-10 was associated with increased prohibitin, thereby ameliorating intestinal fibrosis (40). There has been no study, to the best of our knowledge, which has investigated the potential association of PHB with ECM accumulation in murine alveolar epithelial cells following treatment with LPS. Following previous studies, the present study hypothesized that, in an LPS-induced alveolar epithelial cell injury model, upregulation of PHB expression may effectively alleviate pulmonary fibrosis and become a novel therapeutic target.

Although the mechanism underlying PHB and ECM accumulation in pulmonary fibrosis remains to be elucidated, PHB and ECM components were upregulated in the ALI cell model in the present study. The possible signaling pathway merits investigation in further research into the process of pulmonary fibrosis, which includes pulmonary inflammation, apoptosis and fibrosis.

In conclusion, the present study identified that the PHB expression level increased and ECM components accumulated in murine alveolar epithelial cells with LPS-induced acute injury, and further investigations may be performed to verify the detailed mechanism.

\section{Acknowledgements}

The present study was supported by the Natural Science Foundation of China under grant no. 81400719.

\section{References}

1. Luh SP and Chiang $\mathrm{CH}$ : Acute lung injury/acute respiratory distress syndrome (ALI/ARDS): The mechanism, present strategies and future perspectives of therapies. J Zhejiang Univ Sci B 8: 60-69, 2007.

2. Matthay MA, Ware LB and Zimmerman GA: The acute respiratory distress syndrome. J Clin Invest 122: 2731-2740, 2012.

3. Tomashefski JF Jr: Pulmonary pathology of acute respiratory distress syndrome. Clin Chest Med 21: 435-466, 2000.

4. Ware LB and Matthay MA: The acute respiratory distress syndrome. N Engl J Med 342: 1334-1349, 2000.

5. Orme JJ, Romney JS, Hopkins RO, Pope D, Chan KJ, Thomsen G, Crapo RO and Weaver LK: Pulmonary function and health-related quality of life in survivors of acute respiratory distress syndrome. Am J Respir Crit Care Med 167: 690-694, 2003.

6. Chida M, Ono S, Hoshikawa Y and Kondo T: Subclinical idiopathic pulmonary fibrosis is also a risk factor of postoperative acute respiratory distress syndrome following thoracic surgery. Eur J Cardiothorac Surg 34: 878-881, 2008.

7. Fukuda Y, Ishizaki M, Masuda Y, Kimura G, Kawanami O and Masugi Y: The role of intraalveolar fibrosis in the process of pulmonary structural remodeling in patients with diffuse alveolar damage. Am J Pathol 126: 171-182, 1987.

8. Lin P: Targeting interleukin-6 for noninfectious uveitis. Clin Ophthalmol 9: 1697-1702, 2015.

9. Rose-John S: The soluble interleukin-6 receptor and related proteins. Best Pract Res Clin Endocrinol Metab 29: 787-797, 2015.

10. De Lauretis A, Sestini P, Pantelidis P, Hoyles R, Hansell DM, Goh NS, Zappala CJ, Visca D, Maher TM, Denton CP, et al: Serum interleukin 6 is predictive of early functional decline and mortality in interstitial lung disease associated with systemic sclerosis. J Rheumatol 40: 435-446, 2013. 
11. O'Donoghue RJ, Knight DA, Richards CD, Prêle CM, Lau HL Jarnicki AG, Jones J, Bozinovski S, Vlahos R, Thiem S, et al: Genetic partitioning of interleukin-6 signalling in mice dissociates Stat3 from Smad3-mediated lung fibrosis. EMBO Mol Med 4: 939-951, 2012.

12. Yoshizaki A, Yanaba K, Ogawa A, Asano Y, Kadono T and Sato S: Immunization with DNA topoisomerase I and Freund's complete adjuvant induces skin and lung fibrosis and autoimmunity via interleukin-6 signaling. Arthritis Rheum 63: 3575-3585, 2011.

13. Snedden WA and Fromm H: Characterization of the plant homologue of prohibitin, a gene associated with antiproliferative activity in mammalian cells. Plant Mol Biol 33: 753-756, 1997.

14. Merkwirth $\mathrm{C}$ and Langer T: Prohibitin function within mitochondria: Essential roles for cell proliferation and cristae morphogenesis. Biochim Biophys Acta 1793: 27-32, 2009.

15. Koushyar S, Jiang WG and Dart DA: Unveiling the potential of prohibitin in cancer. Cancer Lett 369: 316-322, 2015.

16. Peng YT, Chen P, Ouyang RY and Song L: Multifaceted role of prohibitin in cell survival and apoptosis. Apoptosis 20 1135-1149, 2015.

17. Sharma A and Qadri A: Vi polysaccharide of Salmonella typhi targets the prohibitin family of molecules in intestinal epithelial cells and suppresses early inflammatory responses. Proc Natl Acad Sci USA 101: 17492-17497, 2004.

18. Theiss AL, Idell RD, Srinivasan S, Klapproth JM, Jones DP, Merlin D and Sitaraman SV: Prohibitin protects against oxidative stress in intestinal epithelial cells. FASEB J 21: 197-206, 2007.

19. Gamble SC, Chotai D, Odontiadis M, Dart DA, Brooke GN, Powell SM, Reebye V, Varela-Carver A, Kawano Y, Waxman J and Bevan CL: Prohibitin, a protein downregulated by androgens, represses androgen receptor activity. Oncogene 26: 1757-1768, 2007.

20. Theiss AL and Sitaraman SV: The role and therapeutic potential of prohibitin in disease. Biochim Biophys Acta 1813: 1137-1143, 2011.

21. Theiss AL, Vijay-Kumar M, Obertone TS, Jones DP, Hansen JM Gewirtz AT, Merlin D and Sitaraman SV: Prohibitin is a nove regulator of antioxidant response that attenuates colonic inflammation in mice. Gastroenterology 137: 199-208, 208.e1-6, 2009.

22. Soulitzis N, Neofytou E, Psarrou M, Anagnostis A Tavernarakis N, Siafakas N and Tzortzaki EG: Downregulation of lung mitochondrial prohibitin in COPD. Respir Med 106: 954-961, 2012

23. Agrawal T, Gupta GK and Agrawal DK: Vitamin D deficiency decreases the expression of VDR and prohibitin in the lungs of mice with allergic airway inflammation. Exp Mol Pathol 93: $74-81,2012$.

24. Ko KS, Tomasi ML, Iglesias-Ara A, French BA, French SW, Ramani K, Lozano JJ, Oh P, He L, Stiles BL, et al: Liver-specific deletion of prohibitin 1 results in spontaneous liver injury, fibrosis and hepatocellular carcinoma in mice. Hepatology 52 : 2096-2108, 2010.

25. Zhou TB, Qin YH, Lei FY, Huang WF and Drummen GP: Prohibitin attenuates oxidative stress and extracellular matrix accumulation in renal interstitial fibrosis disease. PLoS One 8: e77187, 2013
26. Sanchez-Quiles V, Segura V, Bigaud E, He B, O'Malley BW, Santamaría E, Prieto J and Corrales FJ: Prohibitin-1 deficiency promotes inflammation and increases sensitivity to liver injury. J Proteomics 75: 5783-5792, 2012.

27. Hu X, Tian Y, Qu S, Cao Y, Li S, Zhang W, Zhang Z, Zhang N and Fu Y: Protective effect of TM6 on LPS-induced acute lung injury in mice. Sci Rep 7: 572, 2017.

28. Zhao J, He D, Su Y, Berdyshev E, Chun J, Natarajan V and Zhao Y: Lysophosphatidic acid receptor 1 modulates lipopolysaccharide-induced inflammation in alveolar epithelial cells and murine lungs. Am J Physiol Lung Cell Mol Physiol 301: L547-L556, 2011.

29. Livak KJ and Schmittgen TD: Analysis of relative gene expression data using real-time quantitative PCR and the 2(-Delta Delta C (T)) method. Methods 25: 402-408, 2001.

30. Rocco PR, Dos SC and Pelosi P: Lung parenchyma remodeling in acute respiratory distress syndrome. Minerva Anestesiol 75: 730-740, 2009

31. Burnham EL, Janssen WJ, Riches DW, Moss M and Downey GP: The fibroproliferative response in acute respiratory distress syndrome: Mechanisms and clinical significance. Eur Respir J 43: 276-285, 2014.

32. Cottin V: Interstitial lung disease. Eur Respir Rev 22: 26-32, 2013.

33. Chapman HA: Epithelial-mesenchymal interactions in pulmonary fibrosis. Annu Rev Physiol 73: 413-435, 2011.

34. Dong WW, Zhang YQ, Zhu XY, Mao YF, Sun XJ, Liu YJ and Jiang L: Protective effects of hydrogen-rich saline against lipopolysaccharide-induced alveolar epithelial-to-mesenchymal transition and pulmonary fibrosis. Med Sci Monit 23: 2357-2364, 2017.

35. He D, Su Y, Usatyuk PV, Spannhake EW, Kogut P, Solway J, Natarajan V and Zhao Y: Lysophosphatidic acid enhances pulmonary epithelial barrier integrity and protects endotoxin-induced epithelial barrier disruption and lung injury. J Biol Chem 284: 24123-24132, 2009.

36. Zhao D, Ding R, Mao Y, Wang L, Zhang Z and Ma X: Heparin rescues sepsis-associated acute lung injury and lethality through the suppression of inflammatory responses. Inflammation 35: 1825-1832, 2012

37. Zhu T, Wang DX, Zhang W, Liao XQ, Guan X, Bo H, Sun JY, Huang NW, He J, Zhang YK, et al: Andrographolide protects against LPS-induced acute lung injury by inactivation of NF-kappaB. PLoS One 8: e56407, 2013

38. Zhou TB, Qin YH, Li ZY, Xu HL, Zhao YJ and Lei FY: All-trans retinoic acid treatment is associated with prohibitin expression in renal interstitial fibrosis rats. Int J Mol Sci 13: 2769-2782, 2012.

39. Theiss AL, Obertone TS, Merlin D and Sitaraman SV: Interleukin-6 transcriptionally regulates prohibitin expression in intestinal epithelial cells. J Biol Chem 282: 12804-12812, 2007.

40. Yuan C, Chen WX, Zhu JS, Chen NW, Lu YM, Ou YX and Chen HQ: IL-10 treatment is associated with prohibitin expression in the Crohn's disease intestinal fibrosis mouse model. Mediators Inflamm 2013: 617145, 2013. 